\title{
Effect of Erbium doped fiber location on double spacing multi-wavelength Brillouin- Erbium fiber laser performance
}

\begin{abstract}
Two different locations of Erbium Doped Fiber (EDF) in dual ring configuration to generate double frequency shifted of multiwavelength Brillouin Erbium fiber laser (BEFL) have been investigated. The experimental results show that the location of EDF has important roles to determine the number of output Stoke signals and tuning range. Beside the Erbium gain, the Brillouin gain also contributes to the performance of BEFL. By putting the EDFA next to the $\mathrm{BP}$, more Stokes lines with wider tuning range were obtained compare by putting the EDF next to the gain medium was observed.
\end{abstract}

Keyword: Multiwavelength; Brillouin erbium doped fiber laser; Double frequency spacing 